\title{
An Implementation of Error Minimization Data Transmission in OFDM using Modified Convolutional Code
}

\author{
Hendy Briantoro, I Gede Puja Astawa, Amang Sudarsono \\ Politeknik Elektronika Negeri Surabaya \\ Jl. Raya ITS - Kampus ITS Sukolilo, Surabaya 60111, INDONESIA \\ Telp : 6231594 7280, Fax : 62315946114 \\ E-mail: hendy@pens.ac.id, puja@pens.ac.id, amang@pens.ac.id
}

\begin{abstract}
This paper presents about error minimization in OFDM system. In conventional system, usually using channel coding such as BCH Code or Convolutional Code. But, performance BCH Code or Convolutional Code is not good in implementation of OFDM System. Error bits of OFDM system without channel coding is $5.77 \%$. Then, we used convolutional code with code rate $1 / 2$, it can reduce error bitsonly up to $3.85 \%$. So, we proposed OFDM system with Modified Convolutional Code. In this implementation, we used Software Define Radio (SDR), namely Universal Software Radio Peripheral (USRP) NI 2920 as the transmitter and receiver. The result of OFDM system using Modified Convolutional Code with code rate is able recover all character received so can decrease until $0 \%$ error bit. Increasing performance of Modified Convolutional Code is about 1 $\mathrm{dB}$ in BER of $10^{-4}$ from $\mathrm{BCH}$ Code and Convolutional Code. So, performance of Modified Convolutional better than BCH Code or Convolutional Code.
\end{abstract}

Keywords: OFDM, BCH Code, Convolutional Code, Modified Convolutional Code, SDR, USRP

\section{INTRODUCTION}

Orthogonal Frequency Division Multiplexing (OFDM) is multicarrier efficiency bandwidth scheme for digital communication where the frequency of its subcarrier overlaps the other [1]. There has been some researches related to OFDM, whether simulation using MATLAB software or implementation using Field Programmable Gate Array (FPGA) hardware. One of them was conducted in Universidade de Aveiro Portugal [2], the research was about OFDM which implemented in FPGA. This FPGA used Virtex-6 processor. The receiver used was Maximum Likelihood (ML) and Carrier Frequency Offset (CFO) Estimator to reduce the excessive information in Cyclic Prefix (CP). The channel equalization used Zero Forcing and channel estimation used Least Square [2]. However, the implementation of this 
research only used Xilinx System Generator in MATLAB with one FPGA. The data transmission was not done in the real condition from one device to another device but only in one device.

OFDMsystem has data rate faster than single carrier systems because the OFDM signal is split into multiple subcarriers. In addition, there was a researchby Ahmad Zainudin et al [3]. The reasearch was about data transmision use DPSK, DQPSK and GMSK modulation which is implemented usingUniversal Software Radio Peripheral (USRP).Unfortunately, the implementation presented and measured whether data is received succesfully or not, and was not able to recover received data [3].

In order data can be received well, it needsone or more additional techniques to increase data robustness. One of the techniques used is channel coding. There are some types of channel coding such as block code, LDPC code, $\mathrm{BCH}$ code and Convolutional code. A research related to channel coding has been already done by Henry Hendrix [4]. His research implemented Convolutional code and Viterbi Decode using DSK TMS320C55x Board. The drawback of the implementation is that data transmission was not done from one device to another device but only in one device. Only in one DSK TMS320C55x Board.

Due to the lack of the previous researches, this research proposes an implementation of OFDM system using channel coding Modified Convolutional Code in USRP for error minimalization for data transmision. Error bits of OFDM system without channel coding is $5.77 \%$. Then, we used convolutional code with code rate $1 / 2$, it can reduce error bits up to $3.85 \%$. The result of OFDM system using Modified Convolutional Code with same code rate is able recover all character received so can decrease error bits until 5.77\%. Meanwhile in NLoS condition, Modified Convolutional Code can decode all data when using 8 characters. In 12 characters, only there is a error bit or $1.04 \%$ of transmitted data. In 14 characters, there are 2 error bits or $1.79 \%$ of transmitted data. Then, in 16 characters there are 9 error bits or $7.03 \%$ of transmitted data. From the result of the experiment above, it can be analyzed that performance Modified Convolutional Code is enough good in NLoS condition. This research uses NI USRP 2920. This device is supported by FPGA chip. FPGA is programmable device. The programming languages used are VHDL, C, Phyton, and LabView. This device has been completed with $\mathrm{RF}$ front-end module to transmit or receive signal, so user is able to interface radio signal to transmit or receive easily. This is the reason why we use NI USRP 2920. By using this device we can easily implement complex algorithm in our research and able to finish it faster with satisfactory result. We can develop many algorithms but only use one device. It has bandwidth streaming at $25 \mathrm{MS} / \mathrm{s}$ for host-based processing with NI LabVIEW [5]. This paper is organized as follows, firstly we describe the introduction about this research. In second section, we present related work of this research. Then, we explain our proposed system in Section 3. In Section 4, we explain about OFDM system, Convolutional code, and proposed Modified Convolutional Code. Furthermore, we present system implementation in Section 5.Section 
6, we discuss about experimental result. Finally, our conclusion is created in Section 7.

\section{RELATED WORKS}

In Universidade de Aveiro Portugal, there was a research by Tiago Pereiraet al [2]. It was about OFDM which implemented in FPGA. FPGA used was Virtex-6 processor. The receiver used was Maximum Likelihood (ML) and Carrier Frequency Offset (CFO) Estimator to reduce the excessive information in Cyclic Prefix (CP). The channel equalization used Zero Forcing and channel estimation used Least Square. However, the implementation of this research only used Xilinx System Generator in MATLAB with one FPGA. The data transmission was not done in the real condition from one device to another device but only in one device[2].

In addition, there was aresearch by Abirami et al[6] on analyzing the performance of OFDM using Software Define Radio (SDR) and Universal Software Radio Peripheral (USRP) in real time. Here, they use two methods, OFDM simulation in GNU Radio and OFDM in real time.The implementation of OFDM systems uses SISO(Single Input Single Output) [6]. Next, there was a researchby Ahmad Zainudin et al [3]. The reasearch was about data transmision use DPSK, DQPSK and GMSK modulation which is implemented using USRP. The implementation presented and measured whether data is received succesfully or not, and was not able to recover received data [3].

On the other hand, there was a research done by Henry Hendrix [4]. His research is about implementation Convolutional code and Viterbi Decode using DSK TMS320C55x [4]. The drawback of the implementation is that data transmission was not done from one device to another device but only in one device. Only in one DSK TMS320C55x Board.

Then, there was a research done by Irfan Y. Khan et al [7]. They improved performance of OFDM system using Convolutional Code and BCH Code. Their research using adaptive modulation and compare the performance between Convolutional Code and BCH Code. And there was a research done by Anjali Kafaltiya et al [8]. They improved performance of MIMO OFDM using combination BCH Coding and Interleaving.

\section{ORIGINALITY}

In this paper, we proposed channel coding Modified Convolutional code in OFDM system to achieve error minimization data transmision. Modified Convolutional Code is modification from Convolutional Code. Before data is encoded use Convolution Code, data is added with some bits which is generated by $\mathrm{BCH}$ Code with code rate $1 / 2$ algorithm. This research is implemented in real condition using USRP on $910 \mathrm{MHz}$ carrier frequency.

\section{SYSTEM DESIGN}

In An Implementation of Error Minimization Data Transmission in OFDM using Modified Convolutional Code research implement OFDM system, Convolutional Code and Modified Convolutional Code. 


\subsection{Orthogonal Frequency Division Multiplexing (OFDM)}

OFDM is a wireless technology that uses a dividing technique to divide carrier into several subcarriers which are orthogonal one another. Multiple orthogonal sub-carrier signals overlapping the spectrum can be generated by generalizing the single carrier Nyquist criterion into a multi-carrier criterion [9]. Practically, the process of the Discrete Fourier Transform (DFT) and inverse DFT (IDFT) is useful for the implementation of orthogonal signals. DFT and IDFT are efficient when the Fast Fourier Transform (FFT) and Inverse FFT (IFFT) are used. With the overlapping frequency therefore OFDM scheme can save the use of frequency. In OFDM scheme, there is a guard interval in the time domain which is called cyclic prefix (CP). This CP can reduce the inter-symbol interferences (ISI) between OFDM symbols [9]. Figure1 shows a block diagram of the transmitter and receiver in an OFDM system.

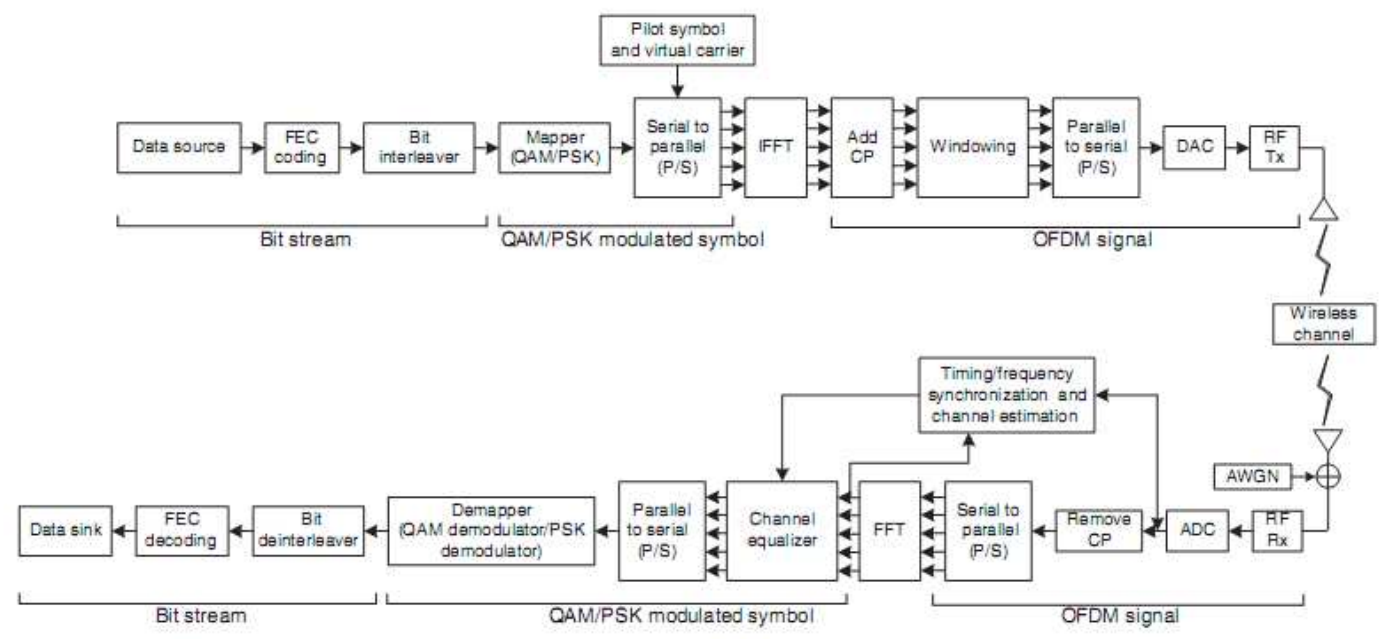

Figure 1. Block diagram of transmitter and receiver OFDM system [9]

IFFT and FFT are the main processes in OFDM system. IFFT is a modulator and FFT is the demodulator. Equation (1) and(2) show the formula of FFT and IFFT, respectively.

FFT:

$x(k)=\sum_{n=0}^{N-1} x(n) \sin \left(\frac{2 \pi k n}{N}\right)+j \sum_{n=0}^{N-1} x(n) \cos \left(\frac{2 \pi k n}{N}\right)$

IFFT :

$x(n)=\sum_{m=0}^{N-1} x(k) \sin \left(\frac{2 \pi k n}{N}\right)+j \sum_{m=0}^{N-1} x(k) \cos \left(\frac{2 \pi k n}{N}\right)$

Where $\mathrm{x}(\mathrm{n})$ is the coeffcients of the sines and cosines of frequency $2 \pi \mathrm{k} / \mathrm{N}$ where $\mathrm{k}$ is the index of the frequencies over the $\mathrm{N}$ frequencies, and $\mathrm{n}$ is the time index, $x(k)$ is the value of the spectrum for the $k^{\text {th }}$ frequency and $x(n)$ is the value of the signal at the time $n$. 
Data transmission in OFDM system is deliveredin parallel. Then, the modulation is performed on each sub-carrier. This modulation can be BPSK, QPSK, QAM or other modulation techniques. Furthermore, the modulated signal has been applied to the Inverse Discrete Fourier Transform (IDFT) such that OFDM symbols can be generated. This allows the use of IDFT frequency allocation mutually perpendicular (orthogonal). After the OFDM symbols are converted into serial, and then the signal is sent [9].

OFDM signal $x(t)$ in interval $\mathrm{mT}_{\mathrm{u}} \leq t \leq(\mathrm{m}+1) \mathrm{T}_{\mathrm{u}}$ can be presented by equation (3).

$$
x(t)=\sum_{k=0}^{N_{q}-1} x_{k}(t)=\sum_{k=0}^{N_{q}-1} a_{k}^{(m)} e^{j 2 \pi k \Delta f t}
$$

With $\mathrm{x}(t)$ is modulate subcarrier k with frequency $f_{k}=k . \Delta f$ and $a_{k}^{(m)}$ is the modulation symbol applied to subcarrier $(\mathrm{k})$ during the interval to the OFDM. Modulation symbols can be BPSK, QPSK, 16QAM or 64QAM [11]. Two orthogonal subcarriers OFDM $x_{k 1}(t)$ and $x_{k 2}(t)$ are presented in equation(4).

$$
\int_{\mathrm{m} \mathrm{T}_{\mathrm{u}}}^{(\mathrm{m}+1) \mathrm{T}_{\mathrm{u}}} \mathrm{x}_{\mathrm{k} 1}(\mathrm{t}) \mathrm{x}_{\mathrm{k} 2}^{*}(\mathrm{t}) \mathrm{dt}=\text { ofor } k_{1} \neq k_{2}
$$

\subsection{Convolutional Code}

Convolutional code is one of channel coding type. Convolutional code uses shift register and XOR gate. There are 2 parameters in convolutional code, they are rate convolutional code (R) and length of memory (K). Rate convolutional code $(\mathrm{R})$ is ratio between bit input and bit output. Rate convolutional code shows in the following equation (5).

$$
R=\frac{K}{n}
$$

Where:

$\mathrm{R}=$ Rate convolutional code

$\mathrm{K}=$ Sum of Bit input

$\mathrm{n}=$ Sum of Bit output

Length of memory $(\mathrm{K})$ is memory with current bit input convolutional code. Length of memory can be defined with the following equation (6).

$$
\mathrm{K}=\mathrm{M}+1
$$

Where:

$\mathrm{K}$ = Length of Memory

$\mathrm{m}=$ Memory

Generator polynomial is impulse respons to register memory of encoder [16]. Block diagram of Convolutional Code with rate $1 / 2$ and $\mathrm{K}=3$ is shown in figure 2 where the generator polynomial is [111 101$]_{2}, R=1 / 2$ and $\mathrm{m}=2$. 


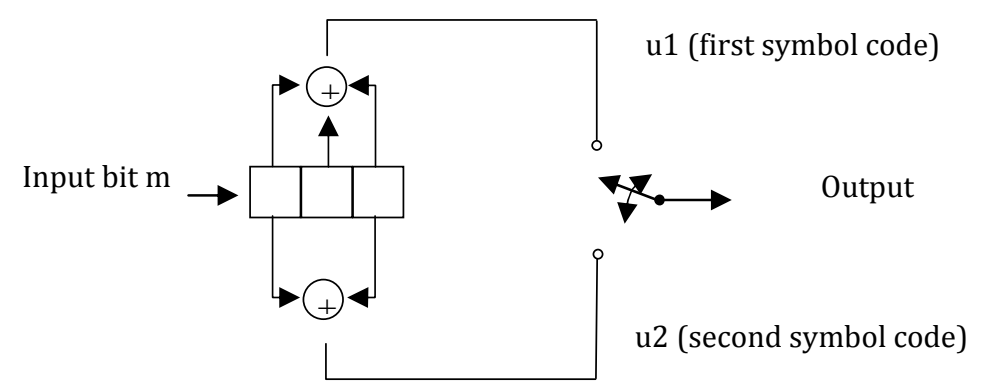

Figure 2. Block diagram of Convolutional Code (rate $1 / 2, K=3$ ) [17]

Viterbi algorithm which implemented is algorithm Maximum Likelihood (ML) and Hard decision showed in Figure 3. Convolutional encoder will code data input, e.g input (11010100). With $1 / 2$ code rate the codeword is $(11,01,01,00,10,00,10,11)$. This codeword transmit to receiver. Codeword mix with noise, the codeword received is $(10,01,01,01,10,00,10,11)$, so it different with transmit codeword.

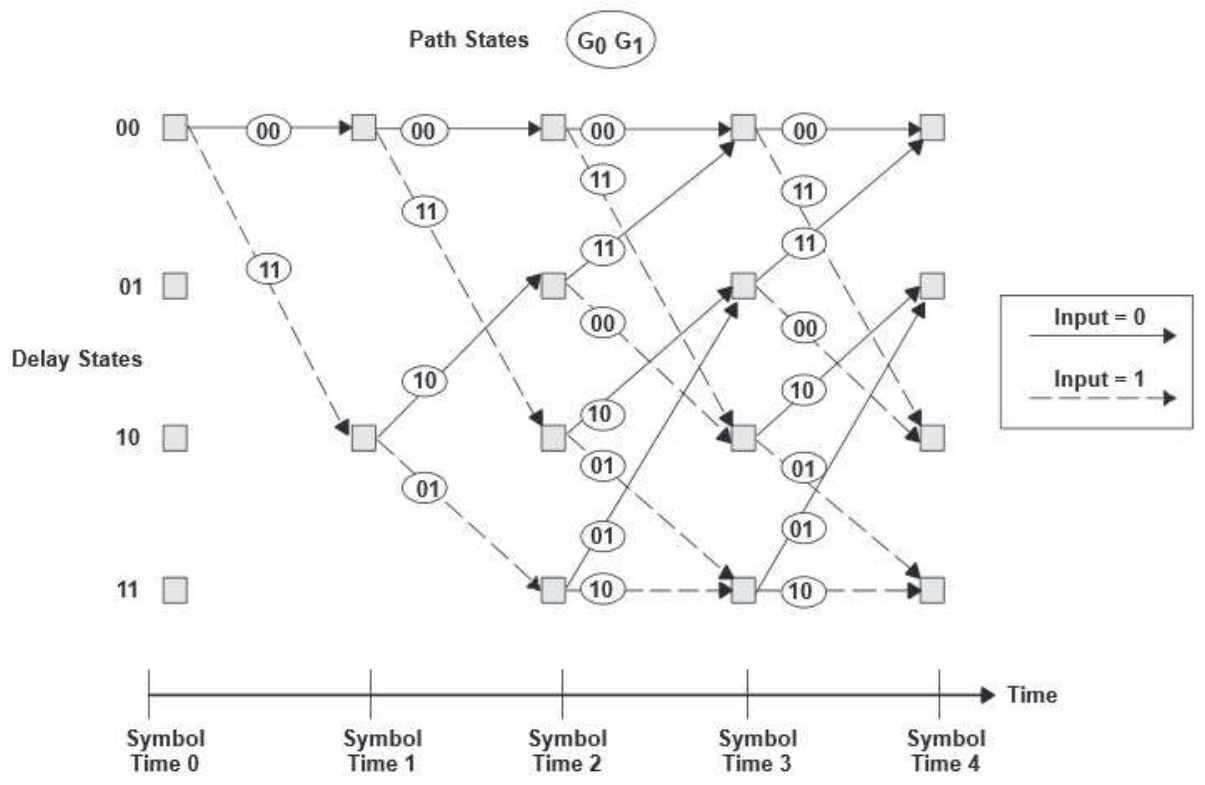

Figure 3. Trellis Diagram of Hard Decision Decoding [4]

The trace back process starts from the concept that each branch (branch) associated with a particular input bits in coding. For example, branching from the state $\mathrm{S} 2$ at $\mathrm{t}=7$ to the state $\mathrm{S} 0$ at $\mathrm{t}=8$ input bits associated with '0' on the coding [4].

\subsection{Proposed Modified Convolutional Code}

As explained in Section 2, channel coding is implemented in both research [7] and [8], BCH Code and Convolutional Code are implemented alternately. The function of channel coding is to reduce error in channel so transmitted symbols is more resistant from impairment or delay in channel. Consequently, we make a new idea so that the error in channel can be solved 
better than previous by combine BCH Code and Convolutional Code and we named it is Modified Convolutional Code. We make a scenario so that signal input is encoded using Modified Convolutional Code. After that the signal is processed by modulator. Block diagram of Modified Convolutional Code is shown in figure 4.

Modified Convolutional Code is proposed channel coding which is implemented in OFDM system. Here is the algorithm of Modified Convolutional code. There are 2 big steps. First, input bit is encoded by proposed algorithm to be first codeword. Then first codeword is encoded by convolutional code algorithm to be final codeword. First codeword length is $n=2^{m}-1$, for $m \in N$ and distance is $d \in N$ and $d \leq(n-1) / 2$. First, determine the minimum polynomial for $\alpha \mathrm{i}$, denoted $\operatorname{mi}(\mathrm{x})$, where $\alpha$ is a primitive root of the unit and $\mathrm{i}=1,2, \ldots, 2 \mathrm{r}$. And then polynomial generator $g(x)$, $g(x)=l c m\left[m_{1}(x), m_{2}(x) \ldots m_{2} r(x)\right]$. Form polinomial $u(x)=x^{n-k} m(x) \bmod$ $g(x)$, where $m(x)=a_{1} x k-1+a_{2} x k-2+\ldots+a k$ is a polynomial corresponding to the message $m=\left(a_{1}, a_{2}, \ldots, a_{k}\right), a_{i} \in Z_{2}$. Thus obtained codeword $\mathrm{c}$ which corresponds to a polynomial $c(x)=x^{n-k} m(x)+u(x)$ [10][11]. Then, first codeword is encoded using default Convolutional Code algorithm. So, the final codeword is generated.

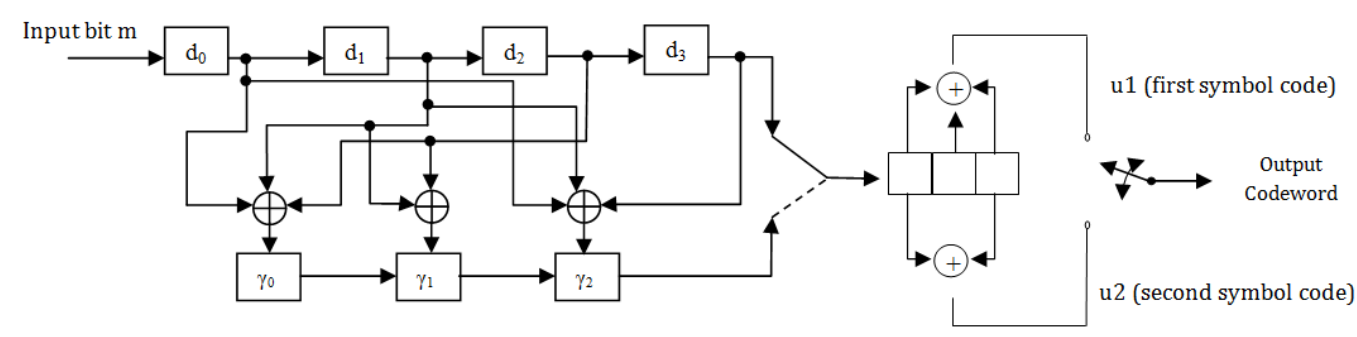

Figure 4. Block diagram of Modified Convolutional Code

\section{System Implementation}

This research implements OFDM system with channel coding Modified Convolutional Code. The system uses NI-USRP N2920 [5] from National Instrument (NI) and employs labView Software [10] to support it. The range of the frequency of NI-USRP 2920 [10] is $50 \mathrm{MHz}$ up to 2,2 GHz. Universal Software Peripheral Radio (USRP) is hardware to support Software Define Radio (SDR) [12][13] in real-time condition. USRP consists of Field Programmable Gate Array (FPGA), Digital to Analog Converter (DAC) and port I/O [14].This research using NI USRP 2920 because it is affordable teaching and research solution. We can develop many algorithms but only use one device. It has bandwidth streaming at $25 \mathrm{MS} / \mathrm{s}$ for host-based processing with NI LabView [5].

This reasearch uses 2 USRPs, oneacts as the transmitter and the other one acts as the receiver. Block diagram of transmitter and receiver are showed in Figure 5 and Figure 6. 


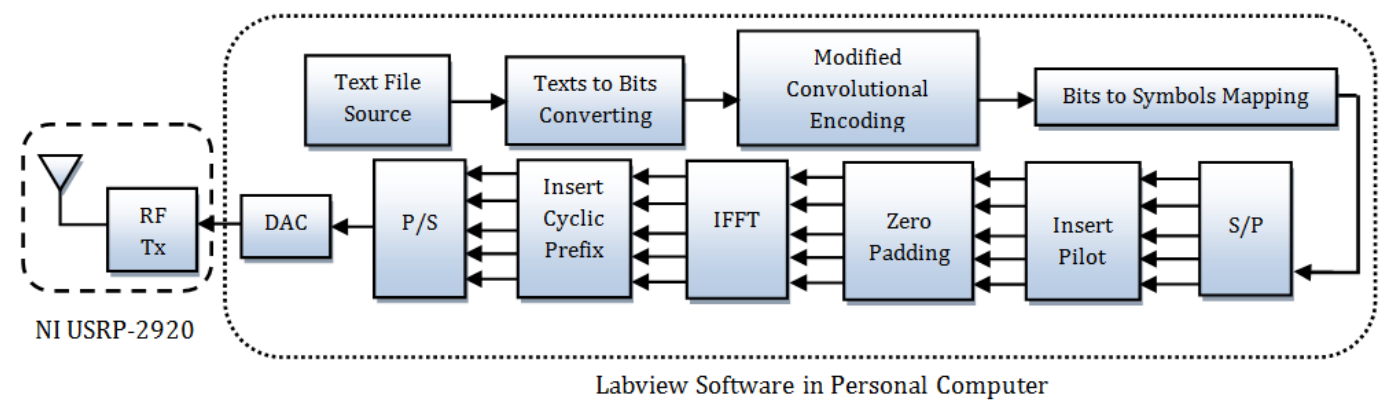

Figure 5. Block diagram of transmitter side

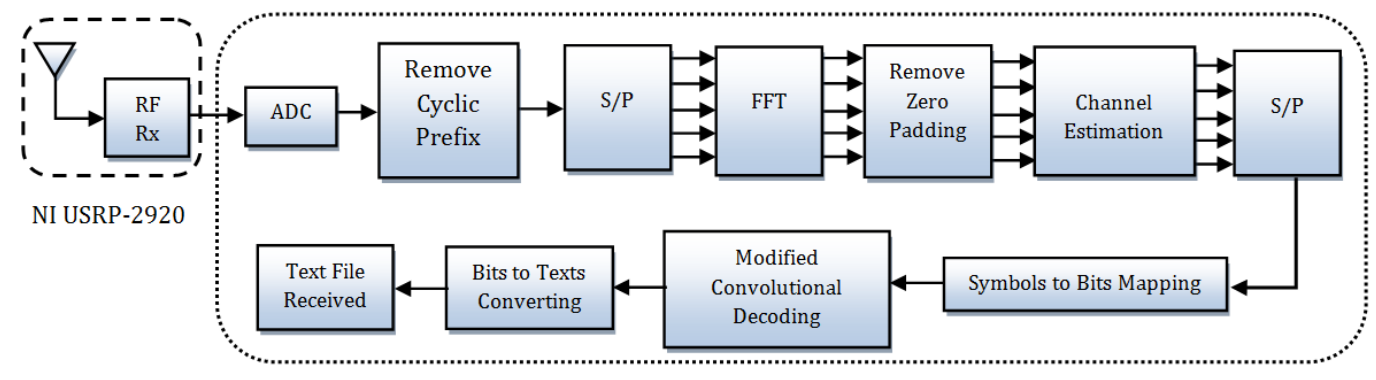

Labview Software in Personal Computer

Figure 6. Block diagram of receiver side

On transmitter side, the first process is determining USRP's IP address and opening the session of USRP hardware. Then, we set the IQ rate and carrier frequency. The next process is reading text file (data) and converting the string data in text file to bits of data. The size of text file is 17 Bytes which is converted into 136 bits of data. Furthermore, bits of data are encoded using Modified Convolutional Code. Encoded bits of data are mapped into symbols. Mapping symbol is determined by the type of modulation technique. There are 3 modulations used in this implementation such as BPSK, 4QAM and 16QAM. There are a bit in BPSK symbols, 2 bits in 4QAM symbol and 4 bits in16QAM symbol. And then, symbols are parallelized. After that, the pilot symbols are inserted OFDM symbols. Size of pilot symbols are 34 bits. Zero padding are added at the edges of the passband ( 43 zeroes as start symbol and 42 zeroes as stop symbol) and 1 zero at DC carrier. OFDM symbols are convert from frequency domain to time domain using Inverse Fast Fourier Transform (IFFT). Then, cyclic prefix are inserted to beginning OFDM symbols. Cyclic prefix from duplicating the last 64 bits of OFDM symbols. Then, OFDM symbols are converted to analog signals and transmitted using USRP.

Analog signals are received by other USRP. Analog signals are converted to digital signals. Then, cylic prefix are removed from signals and divide to be 5 parts. The signals are converted from time domain to frequency domain. Zero padding are removed from OFDM symbols. Pilot symbols are removed and are used as channel estimation reference. After that, OFDM symbols are mapped to bits (demodulation) and decoded using Modified Convolutional decoded. Bits received are converted to file text and compared with source file.The system 
parameters are shown in Table 1.

Table 1. System parameters

\begin{tabular}{|c|c|c|}
\hline \multirow{4}{*}{ Transmitter } & Parameters & Value \\
\cline { 2 - 3 } & Pilot Sequence & 34 symbol pilot \\
\cline { 2 - 3 } & Modulations & BPSK, 4QAM and 16QAM \\
\cline { 2 - 3 } & Number of subcarriers & 256 \\
\cline { 2 - 3 } & FFT size & 256 \\
\hline \multirow{4}{*}{ Guard Intervals } & $\begin{array}{c}\text { 86 bits (43 zeroes at the edges } \\
\text { of the passband and at 1 zero at } \\
\text { DC) }\end{array}$ \\
\cline { 2 - 3 } & Data Rate & $200 \mathrm{KBps}$ \\
\cline { 2 - 3 } & Carrier Frequency & $910 \mathrm{MHz}$ \\
\cline { 2 - 3 } & 1 Frame Data & $320 \mathrm{bit}$ \\
\cline { 2 - 3 } & Cyclic Prefix ratio & $1 / 4$ \\
\cline { 2 - 3 } & Data of symbol OFDM & 136 bit \\
\hline Receiver & Channel Estimator & MMSE \\
\cline { 2 - 3 } & Equalization & Zero Forcing \\
\hline
\end{tabular}

\section{EXPERIMENTALRESULTS AND ANALYSIS}

This experiment uses two Personal Computers (PCs) and two USRPs. One PC and one USRP act as transmitter, other PC and other USRP act as receiver. Figure 7 shows the topology of this experiment.Table 2 shows the hardware specification in our experiment.

Transmitter

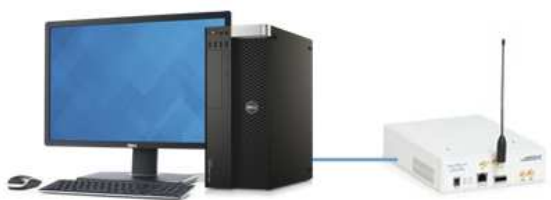

PC 1
USRP 1
Receiver

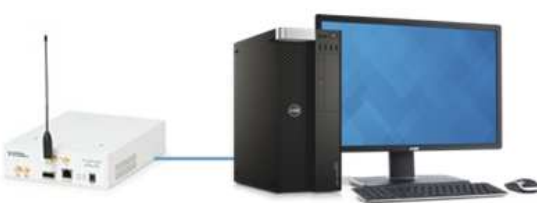

USRP 2

Figure 7. Topology of experiment 
Table 2. Hardware specification in our experiment

\begin{tabular}{|c|c|c|}
\hline No & Hardwares & Specifications \\
\hline \multirow[t]{5}{*}{1} & \multirow{5}{*}{ PC 1 / PC 2} & Processor : Intel ${ }^{\circledR}$ Xeon ${ }^{\circledR}$ Quad Core \\
\hline & & Clock Processor : 3,2 GHz \\
\hline & & Cache : $8 \mathrm{MB}$ \\
\hline & & RAM : 4 GB \\
\hline & & Operating System : Windows 7 64bit \\
\hline \multirow[t]{8}{*}{2} & \multirow[t]{8}{*}{ USRP 1 / USRP 2} & Type : NI-USRP 2920 \\
\hline & & Range Frequency : $50 \mathrm{MHz}$ to 2,2 GHz \\
\hline & & Antenna Frequency : $900 \mathrm{MHz}$ \\
\hline & & $\begin{array}{l}\text { Maximum Output Power } \\
\text { - } 50 \mathrm{MHz} \text { to } 1,2 \mathrm{GHz}: 50 \mathrm{~mW} \text { to } 100 \mathrm{~mW} \\
(17 \mathrm{dBm} \text { to } 20 \mathrm{dBm}) \\
\text { - } 1.2 \mathrm{GHz} \text { to } 2,2 \mathrm{GHz}: 30 \mathrm{~mW} \text { to } 70 \mathrm{~mW} \\
(15 \mathrm{dBm} \text { to } 18 \mathrm{dBm})\end{array}$ \\
\hline & & Gain range : $0 \mathrm{~dB}$ to $31 \mathrm{~dB}$ \\
\hline & & $\begin{array}{l}\text { Maximum I/Q sampling rate : } \\
\text { - } 16 \text {-bit sample width : } 25 \mathrm{MS} / \mathrm{s} \\
\text { - } \quad \text { 8-bit sample width : } 50 \mathrm{MS} / \mathrm{s}\end{array}$ \\
\hline & & DAC : 2 channels, $400 \mathrm{MS} / \mathrm{s}, 16$ bit \\
\hline & & Software support : LabView 2013 \\
\hline
\end{tabular}

The experiment is done in Digital Signal Processing (DSP) Laboratory PENS. Dimension of DSP Laboratory is $8 \mathrm{~m}^{2}$. Topology of DSP Laboratory's environment is showed in Figure 8.

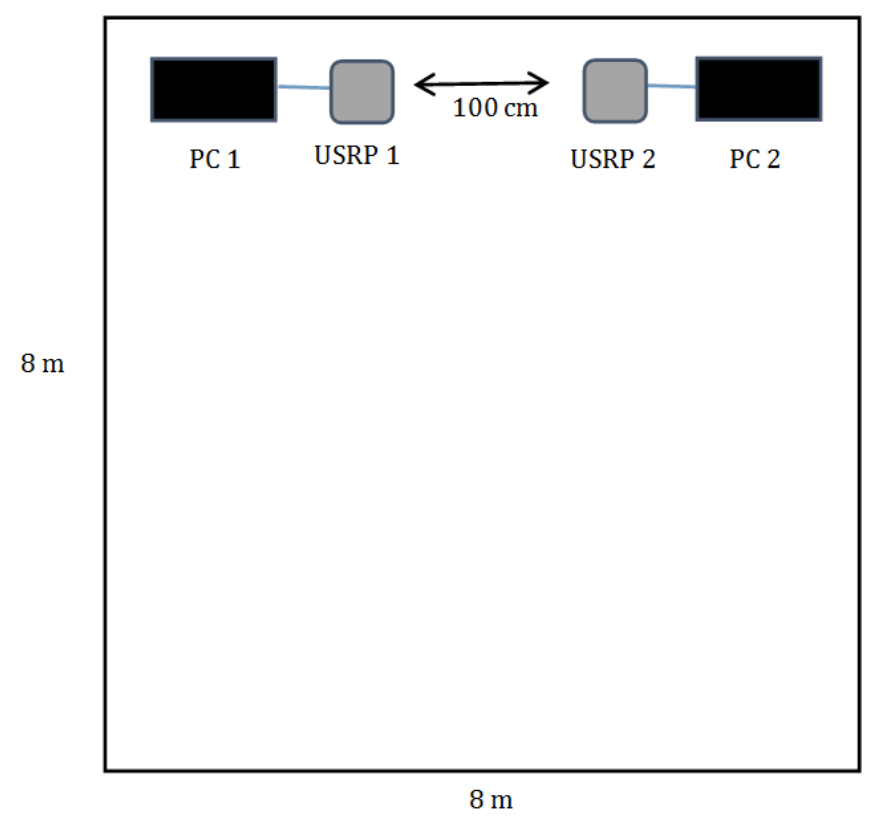

Figure 8. Topology of DSP Laboratory's environment 
There are some experiment scenarios in this research. They are measuring of error bit of OFDM system without Channel Coding, with Convolutional Code, and Modified Convolutional Code, measuringerror bit of OFDM system using Convolutional code with various number of characters, and measuringerror bit of OFDM system using Modified Convolutional code with various number of characters.

\subsection{Error Bit of Data transmission without Channel Coding, with ConvolutionalCode and Modified Convolutional Code Measurement}

First experiment, transmision data use OFDM with channel coding Convolutional Code and Modified Convolutional Code. Transmited data are text file 13 bytes. Data is converted to bits data. Data is transmitted use 4QAM modulation. We useConvolutional code with code rate $1 / 2$ and Modifed Convolutional code with code rate $1 / 2$. This result is shown in Figure 9.

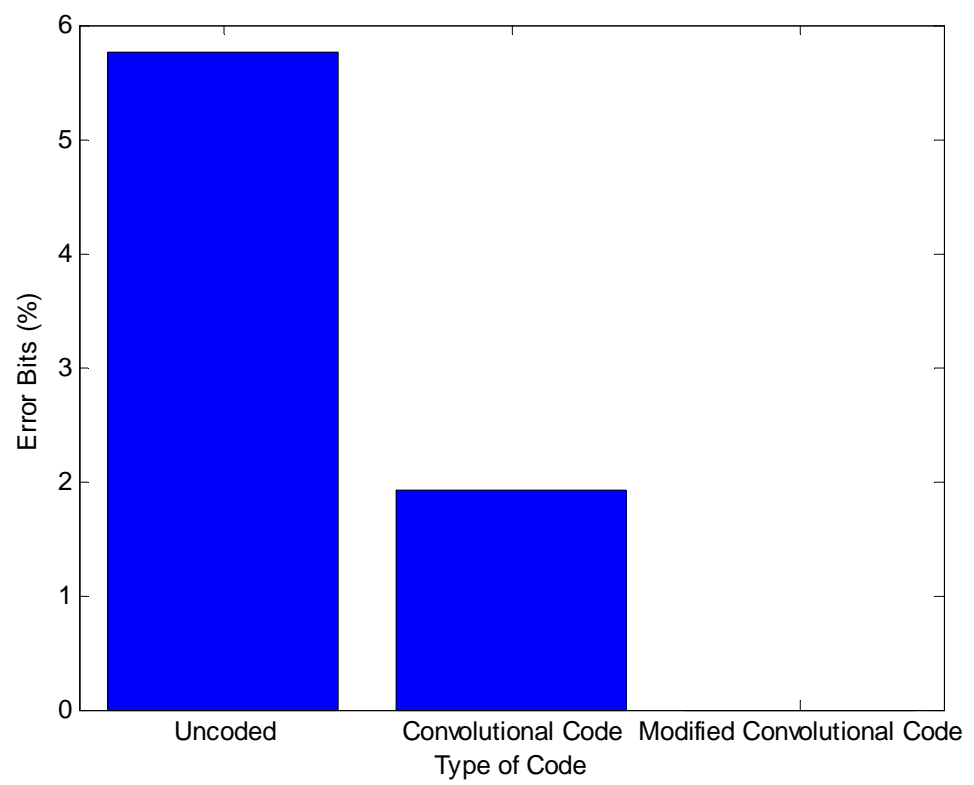

Figure 9.Error bits of Data Received without Code, Convolutional Code, and Modified Convolutional Code

The result of OFDM system using Convolutional Code with code rate $1 / 2$ raised 2 bits error and can decrease error bits 3.85\%. However, when using Modified Convolutional Code with same code rate all character can be received $100 \%$ successful and can decrease error bits until $5.77 \%$. Performance of Modified Convolutional Code is better than Convolutional Code. Increase performance up to $1.92 \%$ 


\subsection{Error Bit of Data transmission using Convolutional Code with Various Number of Characters}

In the second experiment, transmision data use OFDM with channel coding Convolutional Code. This experiment uses some various number of characters, they are 8 characters, 12 characters, 14 characters and 16 characters. Data is converted to bits data. Data is transmitted use 4QAM modulation. We employed Convolutional Code with code rate 3/4. The result is showed in Figure 10.

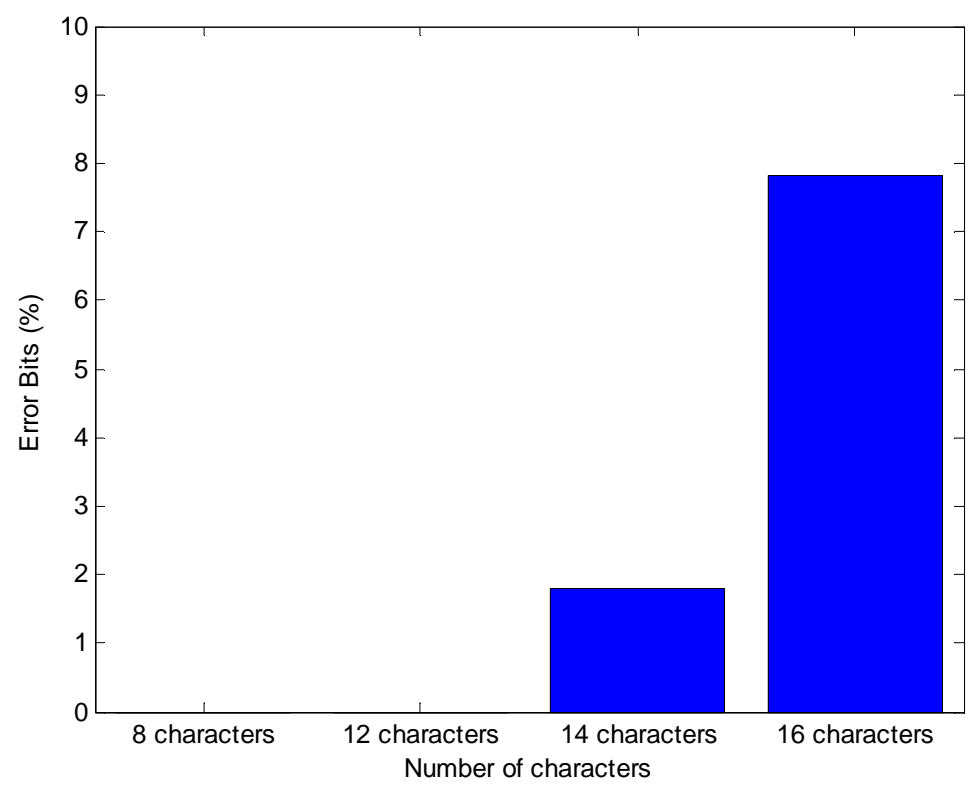

Figure 10. Error bits of data received using Convolutional code with various number of characters

Convolutional code with code rate $3 / 4$ is able to decode all data when using 8 and 12 characters. In 14 characters, there are 2 error bits or $1.79 \%$ of transmitted data. Then, in 16 characters there are 10 error bits or $7.81 \%$ of transmitted data, this error is large enough. For 16 characters or more, the error is more. So, Convolutional code is only able to send character under 16.

\subsection{Error Bit of Data transmission using Modified Convolutional Code with Various Number of Characters}

The third experiment, transmision data use OFDM with Modified Convolutional Code. This experiment uses some various number of characters, they are 8 characters, 12 characters, 14 characters and 16 characters. Data is converted to bits data. Data is transmitted use 4QAM modulation. We employed Modified Convolutional Code with code rate 3/4. The result is showed in Figure 11. 


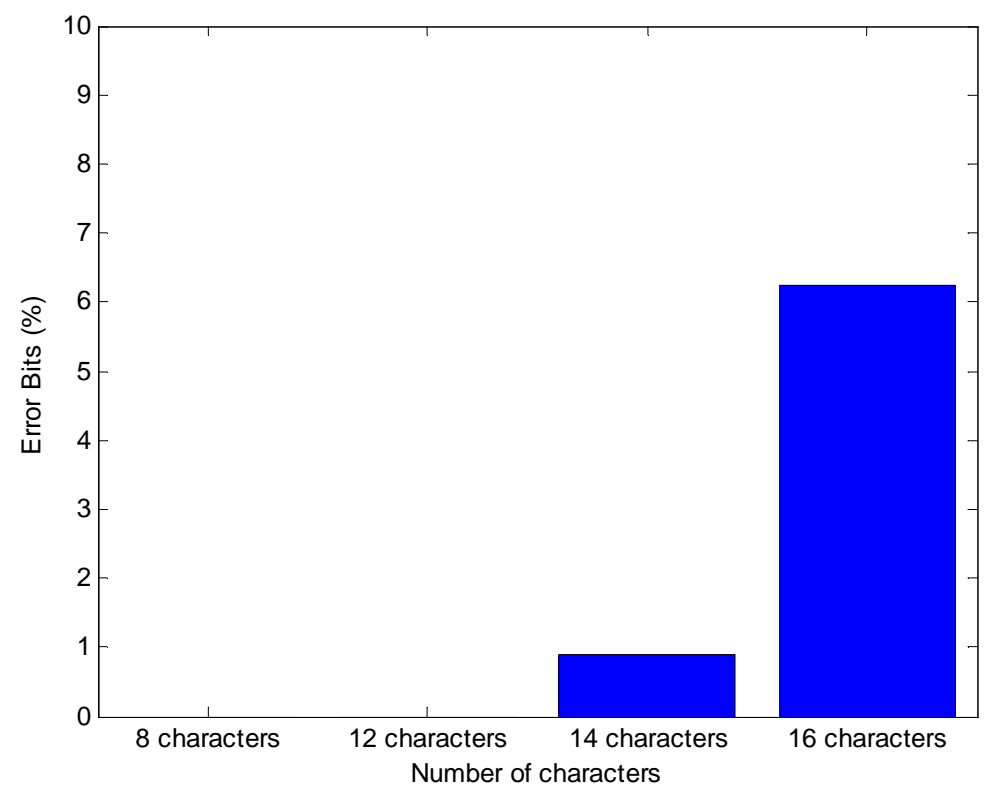

Figure 11. Error bits of data received using Modified Convolutional code with various number of characters

Modified Convolutional code with code rate $3 / 4$ is able to decode all data when using 8 and 12 characters. In 14 characters, there are 1 error bits or $0.89 \%$ of transmitted data. Then, in 16 characters there are 8 error bits or $6.25 \%$ of transmitted data, this error is large enough. For 16 characters or more, the error is more. So, Modified Convolutional Code is only able to send character under 16.From the result of the experiment above, it can be analyzed that the Modified Convolutional Code is better than Convolutional Code.

\subsection{Error Bit of Data transmission using Modified Convolutional Code in LoS and NLoS condition}

The fourth experiment, transmision data use OFDM with channel coding Modified Convolutional Code. This experiment is did in Line of Sight (LoS) condition and Non Line of Sight (NLoS) condition. In LoS condition, distance between USRP 1 and USRP 2 is $100 \mathrm{~cm}$ and no obstacle. In NLoS condition, distance between USRP 1 and USRP 2 is $100 \mathrm{~cm}$ and there is a obstacle which its dimensions is $800 \mathrm{~cm} \times 300 \mathrm{~cm} \times 20 \mathrm{~cm}$ (length $\mathrm{x}$ height $\mathrm{x}$ thickness). This experiment uses some various number of characters, they are 8 characters, 12 characters, 14 characters and 16 characters. Data is converted to bits data. Data is transmitted use 4QAM modulation. We employed Convolutional Code with code rate $1 / 2$. The result is showed in Figure 12. 


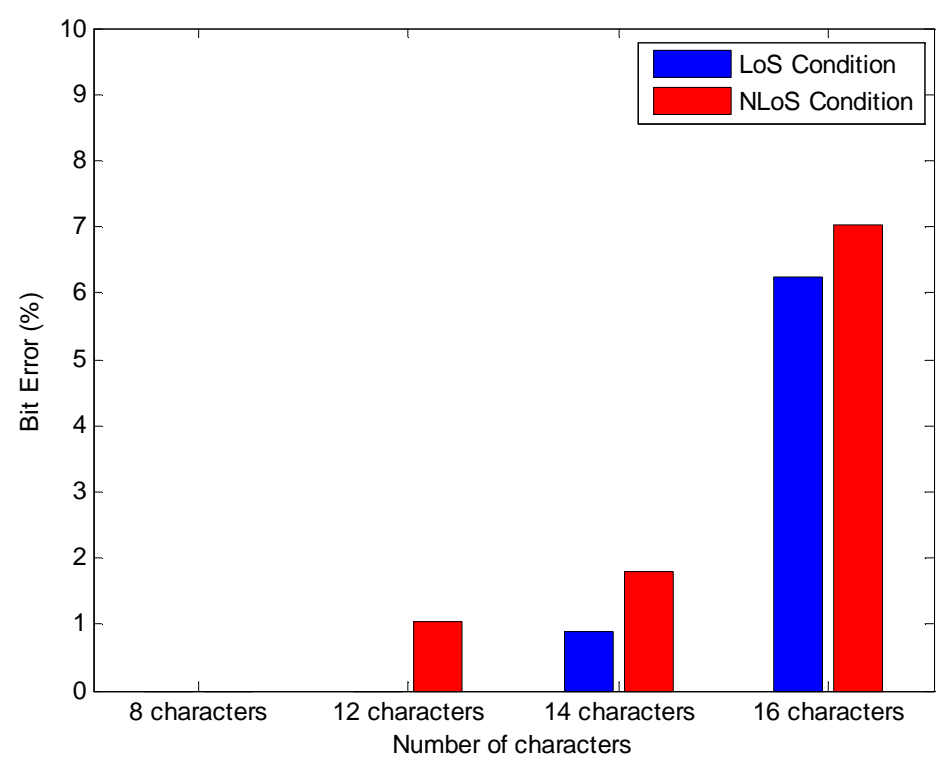

Figure 12. Error bits of data received using Modified Convolutional code in LoS condition and with obstacle condition

In LoS condition, Modified Convolutional Code is able to decode all data when using 8 and 12 characters. In 14 characters, there is a error bit or $0.89 \%$ of transmitted data. Then, in 16 characters there are 8 error bits or $6.25 \%$ of transmitted data. Meanwhile in NLoS condition, Modified Convolutional Code can decode all data when using 8 characters. In 12 characters, only there is a error bit or $1.04 \%$ of transmitted data. In 14 characters, there are 2 error bits or $1.79 \%$ of transmitted data. Then, in 16 characters there are 9 error bits or $7.03 \%$ of transmitted data. From the result of the experiment above, it can be analyzed that performance Modified Convolutional Code is enough good in NLoS condition.

\subsection{Performance BER vs SNR Data Transmission using BCH Code, Convolutional Code and Modified Convolutional Code}

The last experiment, we measure performance BER vs SNR of data transmision. Three channels coding are implemented in this experiment, they are BCH Code, Convolutional Code and Modified Convolutional Code. This experiment uses 128 characters data which are converted be 1024 bits. Then data is sent with $4 Q A M$ modulation. This experiment is did in various Signal to Noise Ratio (SNR) value from $0 \mathrm{~dB}$ until $10 \mathrm{~dB}$. The result is showed in Figure 13. 


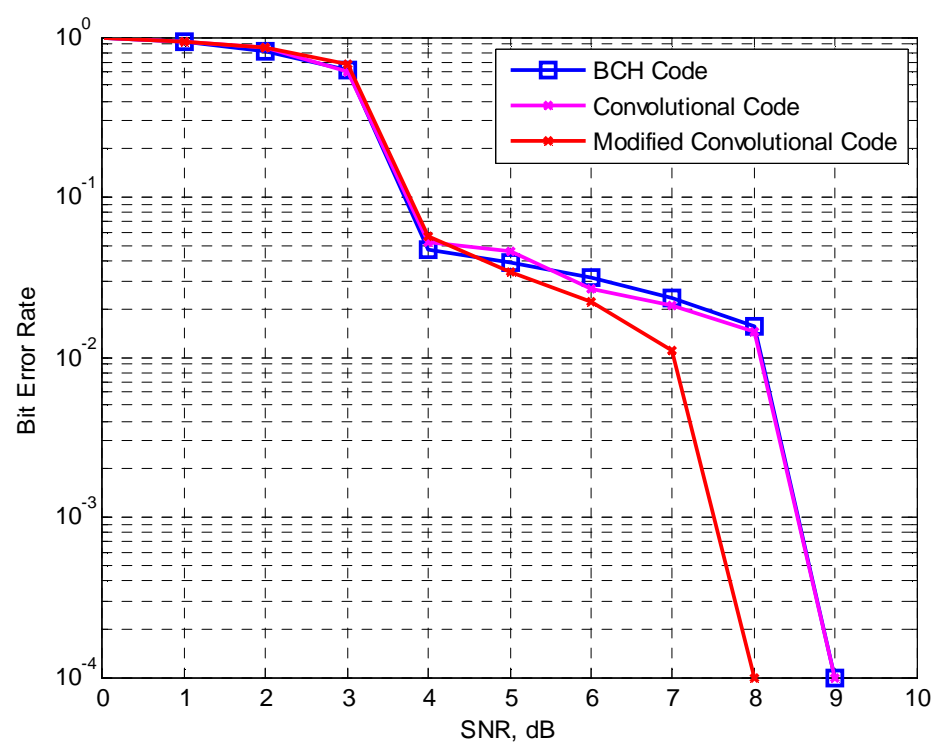

Figure 13. Performance of Data Transmision using BCH Code, Convolutional Code and Modified Convolutional Code

In $0 \mathrm{~dB}$, received data is corrupt. In $3 \mathrm{~dB}, \mathrm{BCH}$ code can decrease BER be 0.9375, Convolutional Code can decrease BER be 0.929 and Modified Convolutional Code can decrease BER be 0.946. When $7 \mathrm{~dB}$, Modified Convolutional Code can decrease BER be 0.011, this BER is better than BCH Code or Convolutional Code and make improvement until 0.0124. BCH Code and Convolutional Code can reduce almost all error in $9 \mathrm{~dB}$ but Modified Convolutional Code can reduce almost all error in $8 \mathrm{~dB}$. Performance of Modified Convolutional better than BCH Code or Convolutional Code. Modified Convolutional can reduce until 0.0154 in same SNR value.

\section{CONCLUSION}

This paper present the implementation of OFDM using Modified Convolutional Code to reduce error of data received. Error bits of received data without code is $5.77 \%$. Convolutional code with code rate $1 / 2$ can decrease error bits of received data become $3.85 \%$ but Modified Convolutional code with same code rate can decrease error bits of received data become $0 \%$, all received data can be recovered. Modified Convolutional Code is better than Convolutional Code because Modified Convolutional Code can recover received data more than Convolutional code. Meanwhile in NLoS condition, Modified Convolutional Code can decode all data when using 8 characters. In 12 characters, only there is a error bit or $1.04 \%$ of transmitted data. In 14 characters, there are 2 error bits or $1.79 \%$ of transmitted data. Then, in 16 characters there are 9 error bits or $7.03 \%$ of transmitted data. From the result of the experiment above, it can be analyzed that performance Modified Convolutional Code is enough good in NLoS condition. BCH Code and Convolutional Code can reduce almost all error in $9 \mathrm{~dB}$ but Modified 
Convolutional Code can reduce almost all error in $8 \mathrm{~dB}$. Performance of Modified Convolutional better than BCH Code or Convolutional Code. Modified Convolutional can reduce until 0.0154 in same SNR value. The next research will implement channel coding in MIMO OFDM system to minimize error OFDM system and use USRP.

\section{REFERENCES}

[1] Gordon L., Stüber, John R. Barry, Steve W. Mclaughlin, Ye(Geoffrey) Li, MaryAnn Ingram, and Thomas G. Pratt, Broadband MIMO-OFDM Wireless Communication, Proceedings of the IEEE, Vol.92 ,No.2, February, 2004

[2] Tiago Pereira, Manuel Violas, João Lourenço, Atílio Gameiro, and Adão Silva, An FPGA Implementation of OFDM Transceiver for LTE Applications, International Journal on Advances in Systems and Measurements,Vol 6 - No 1 \& 2, 2013

[3] Zainuddin, Ahmad, Amang Sudarsono, I Gede Puja Astawa, Reliability Analysis of Digital Communication for Various Data Types Transmision Using GNU Radio and USRP, Industrial Electronic Seminar, 2013

[4] Henry Hendrix, Viterbi Decoding Techniques for the TMS320C55x DSP Generation, April, 2009

[5] National Instrument, An Introduction to Software Define Radio With LabVIEW Communications System Design Software and NI USRP, USA, 2015

[6] Abirami M, Gandhiraj R, Soman K P, Performance Analysis of Real Time OFDM Based Communication System Using GNU Radio and USRP, International Journal of Advanced Research in Computer Science and Software Engineering, Volume 3, Issue 6, June 2013, ISSN: 2277 $128 \mathrm{X}$

[7] Khan, Irfan Y., Sunil U. Nyati, and Umesh S. Bhadade, To Improve Performance of OFDM System Using Optimize Adaptive Coding Technique with Convolutional and BCH Coding, International Journal of Application or Innovation in Engineering \& Management (IJAIEM)Vol 3 - Issue 5, 2014

[8] Kafaltiya, Anjali, and P. S. Sharma, Performance Improvement in MIMO-OFDM using BCH Coding and Interleaving, International Journal of Computer Applications Vol 97 - No 2, 2014.

[9] Yong Soo Cho, Jaekwon Kim, Won Young Yang, Chung-Gu Kang, MIMOOFDM Wireless Communications With MATLAB, Wiley, IEEE Press, 2010.

[10] Ho Shan-Yuan, Daniel J. Kleitman, An Odd Kind of BCH Code, Elsevier, 2012 
[11] Reviriegoa P., C. Argyridesb, J. A. Maestroa, Efficient error detection in Double Error Correction BCH codes for memory applications, Elsevier, 2012

[12] R. Gandhiraja, Ranjini Ramb, K. P. Soman, Analog and Digital Modulation Toolkit for Software Defined Radio, Amrita Vishwa Vidyapeetham (Amrita University), India, 2012.

[13] Waqar Aziz, Ghulam Abbas, Ebtisam Ahmed, Saqib Saleem and Qamarul-Islam, Design Analysis of Analog Data Reception Using GNU Radio Companion (GRC), World Applied Sciences Journal 17 (1): 29-35, ISSN 1818-4952, IDOSI Publications, 2012

[14] Elizabeth A. Thompson, Natha Clem, Isaac Renninger, Timothy Loos, Software-defined GPS receiver on USRP-platform, Purdue University, USA, 2011. 